\title{
MEMS based Hydrogen Sulphide gas detection sensor using Tugsten Oxide Nano fibers
}

\author{
Sauvik Das Gupta ${ }^{1}$, Soumyajit Routh ${ }^{2}$, Subhajit Ghosh $^{3}$, Raquib Buksh ${ }^{4}$, Saurav \\ Debnath $^{5}$, Abhishek Mallik ${ }^{6}$ \\ ${ }^{1,6}$ School of Electrical and Computer Engineering, Oklahoma State University, Stillwater, OK, USA \\ 2,3,4,5 West Bengal University of Technology, Kolkata, West Bengal, India
}

\begin{abstract}
In this paper a semiconductor type MEMS based Hydrogen sulphide gas sensor is proposed. The development of the sensor is done using micromachining and micro fabrication techniques. The major sensing element proposed is tungsten oxidenano-fibers for better enhancement in sensitivity. The synthesis of the nanofibers can bedone using the electro spinning technique. The sensor design can be validated using the Intellisuite software using its various CAD modules. The sensor has various applications such as in oil and gas industry.
\end{abstract}

Keywords: MEMS, micromachining, tungsten oxide nanofibres.

\section{Introduction:}

Hydrogen Sulphide gas also known as "sewer gas" is a very toxic and flammable gas. At low levels hydrogen sulphide gas has a rotten egg odor. At higher levels the nose of the smelling person can become overwhelmed by the gas and he cannot smell it. (1). There is deadliness associated with the hydrogen sulphide gas. The $\mathrm{H}_{2} \mathrm{~S}$ gas is so toxic that its toxicity level is reached before the flammable level is reached. According to the occupational safety and health Administration (US department of labor) the acceptable concentration of the gas in USA is $20 \mathrm{ppm}$ for a 8 hour period and $50 \mathrm{ppm}$ for a 10 hour period. This gas can cause instant death at very high concentration and constant inhaling at low levels may also cause serious damage and conjunctivitis, blurred vision, corneal bullae etc.

Concentration on the oil and gas industry we also see the danger of $\mathrm{H}_{2} \mathrm{~S}$ on Rigs. Sour gas consisting of Hydrogen sulphide exists all over the industrial area. There are some potential hazard areas: On HVAC air vents of accommodation buildings and personnel areas, Driller stand, the shale shaker and bell nipple, Mud return line receiver tank, Crude oil storage tanks, pipes, flanges and valves, remote well sites at the well heads, the storage tanks and flare stacks, in enclosed analyzer building. (2)

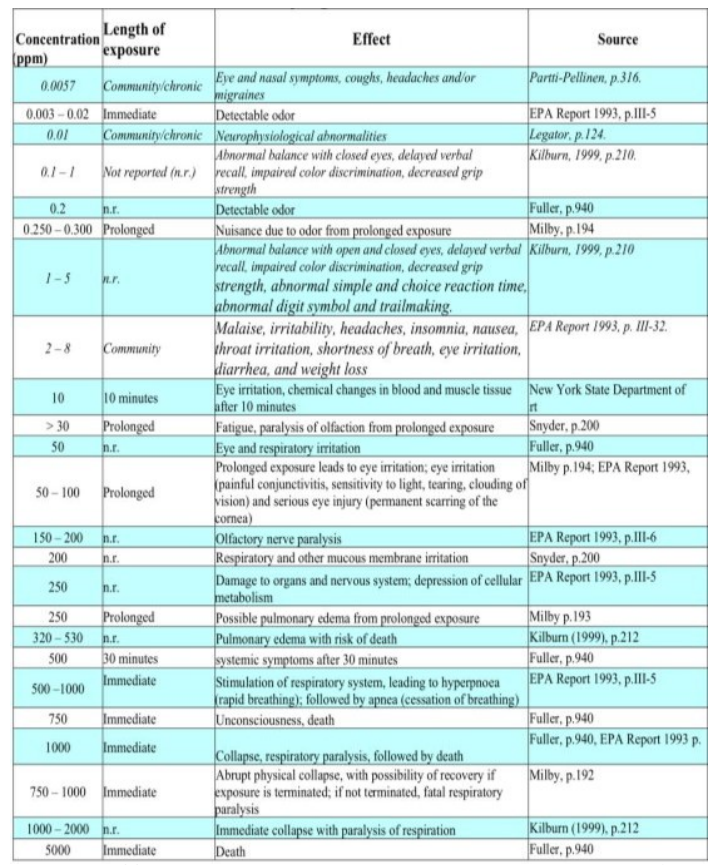

Table 1: Table showing the effect on the person to the corresponding concentration of $\mathrm{H}_{2} \mathrm{~S}$. (3) 


\section{Sensor Facets and design:}

A semiconductor type metal oxide can respond to the presence of a specific gas with a change of its electronic conductivity (4). Metal oxides such as $\mathrm{WO}_{3}, \mathrm{SnO}_{2}, \mathrm{TiO}_{2}$ is can be used.

In this paper Tungsten Oxide $\left(\mathrm{WO}_{3}\right)$ nanofibers is proposed for enhancing the sensitivity of the sensor. It is the main sensing element here.

The core of the sensor consists of the main sensing element. The H2S sensor can be designed in a planar configuration. Pt heater and temperature detector can be deposited onto a support layer of silicon nitride covering the silicon dioxide. It will help to achieve the working temperature of the device. A layer of phosphosilicate glass will be deposited over the heater to separate the sensing and the heating elements. The backside of the substrate below the sensing element should be removed to reduce the power consumption of the heater since silicon acts as a heat sink.

All the MEMS related technologies such as sputtering, photolithography, thin film deposition and etc can be used to fabricate the sensor (8).

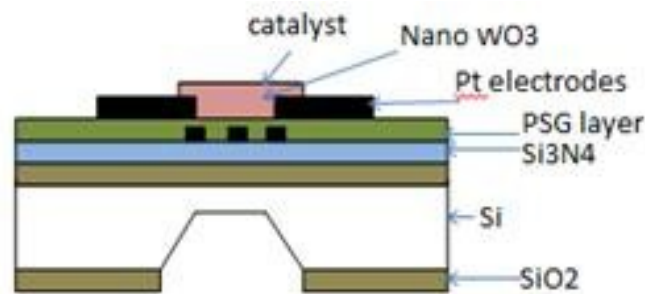

Figure 1: Schematic diagram of the proposed sensor

\section{III. $\mathrm{WO}_{3}$ nanofiber synthesis using electro-spinning:}

There has been a lot of research going on the synthesis of various 1 dimensional nanoscale materials. Among the various materials a lot of focus is being given on the synthesis of $\mathrm{WO}_{3}$ nanomaterials because of its eletrochromic and photochromic properties as well as application in gas sensing and optical properties.(5)

There are various techniques for the synthesis. One of the very effective techniques is electro spinning. A variety of materials such as polymers, inorganic and hybrid compounds nanofibres can be prepared using this technique. In electro spinning a solid fiber is generated as the electrified jet is continuously stretched due to the electrostatic repulsions between the surface charges and the evaporation of the element.

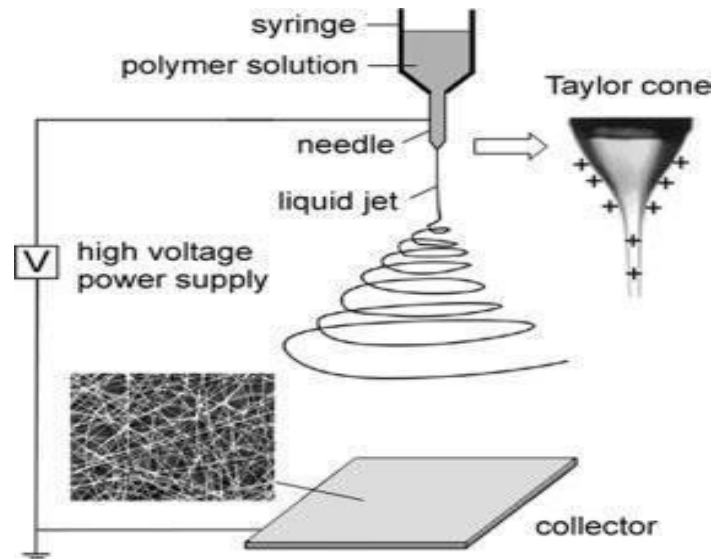

Figure 2: Schematic diagram of electrospinning setup. (6)

A solution of metallic tungsten powder and hydrogen peroxide in proper proportions can be used. Magnetic stirring will be required. Then this solution will be fed into the syringe equipped with needle made of copper. The needle will be connected to a high voltage power supply. A piece of flat aluminum foil can be used to collect the nanofibres. Annealing of the obtained electro spinning fibers film in the air is used to prepare $\mathrm{WO}_{3}$ nanofibres.(7)

\section{Sensor Fabrication:}

Firstly the photolithographic masks will be designed. These images will be transferred to the glass plate using masking material.

A layer of silicon dioxide can be grown on both sides of the cleaned Si wafer by wet thermal oxidation. 
Then a low stress silicon nitride layer will be deposited using Low Pressure Chemical Vapor Deposition. It has high strength, thermal stability and relatively low stress. Then the platinum heater will be deposited and RTD. Lift of process is required for the Pt layer construction. Titanium will be given between the platinum and $\mathrm{Si}_{3} \mathrm{~N}_{4}$ which acts as adhesive. Next a PSG insulation layer was deposited using Atmospheric Vapor Chemical Vapor Deposition (APCVD). Next the Platinum electrodes were deposited using the liftoff technique. Then the synthesized WO3 nano-fibers will be deposited over the Pt layer using the RF sputtering technique. A metal mask can be employed for the patterning of the $\mathrm{WO}_{3}$. The noble metals can be then deposited as catalyst. Lastly the windows at the backside of the sensor will be etched by ap plying and patterning photoresist. The wafers will be then diced and the chips will be bonded with Pt wires and pads. (8)

Step1: Wet Oxidation for Silicon Oxide

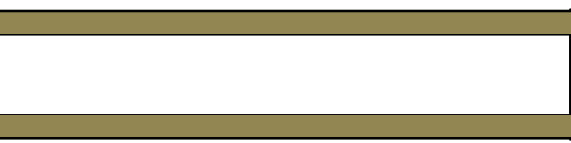

Step2: $\mathrm{LPCVD} \mathrm{Si}_{3} \mathrm{~N}_{4}$ deposition

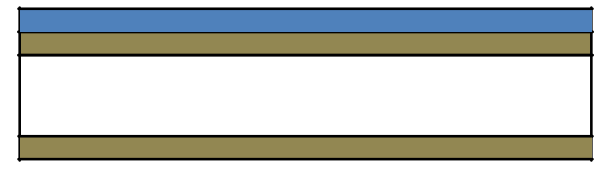

Step3: Heater/Temp detector deposition

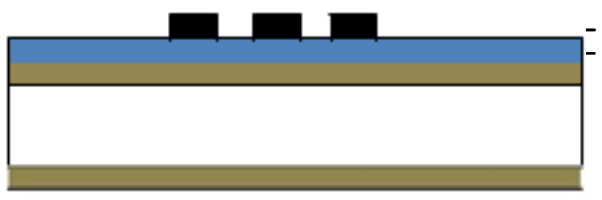

Step4: APCVD PSG Insulation layer

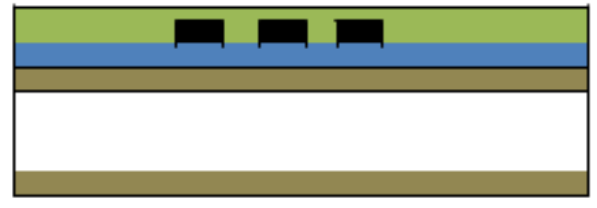

Step5: Pt Electrode Deposition

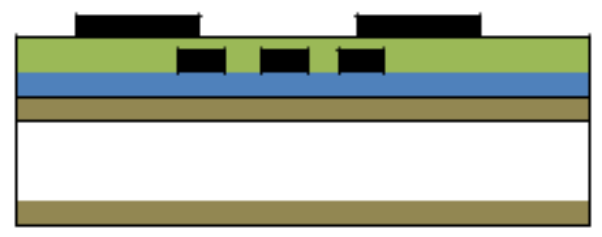

Step6: Nano $\mathrm{WO}_{3}$ deposition

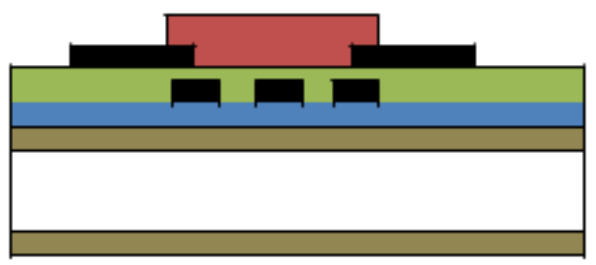


Step7: Catalyst Deposition

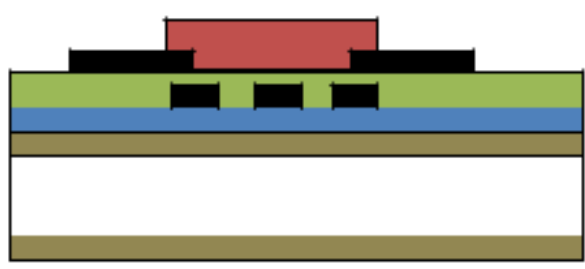

Step 8: Silicon Backside etching

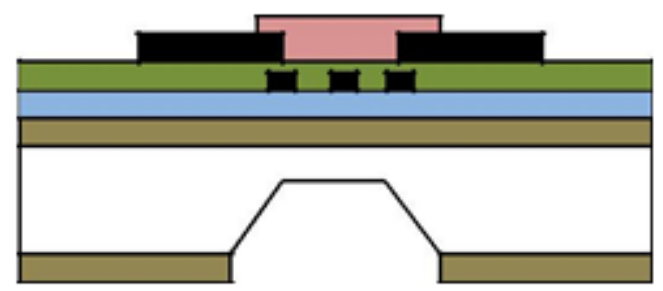

Figure 3: Schematic diagram of the fabrication process

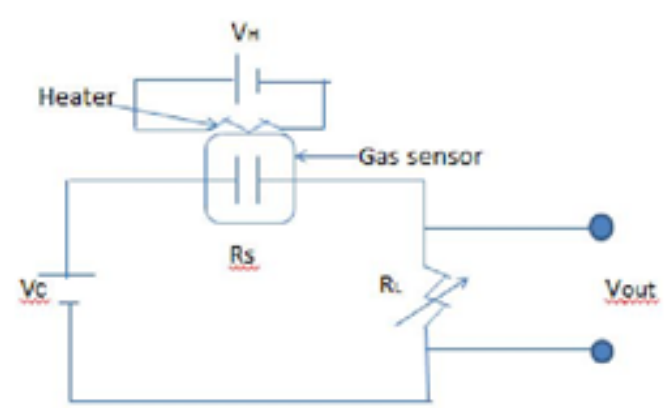

$\mathrm{V}_{\text {out }}=\mathrm{V}_{c} \times\left[\mathrm{RL}_{\mathrm{L}} /(\mathrm{RL}+\mathrm{Rs})\right]$

Figure 4: Circuit of the sensor

\section{Software Simulation:}

The design of the sensor can be performed using various CAD models of Intellisuite software, CAD tool for MEMS design. For the fabrication pr

ocess IntelliFab module can be used and for the sensor circuitry Synple module will be preferable. Then the analysis can be done using the analysis modules.

\section{Conclusion:}

Micro sensors are now being used in various industries using oil and Gas, automobile etc. Various industry professionals were interviewed and the necessity of the MEMS based $\mathrm{H}_{2} \mathrm{~S}$ gas sensor was noted. The fabrication process was studied in detail and the enhancement of the sensing by introduction of the Nano fibers was done.

As a future work there is always a desire to fabricate the sensor.

\section{References:}

[1] Pat Quinn, Governor and Damon T. Arnold, M.D,M.P.H.,Director, Illinois Department of Public health. Environmental Health Fact Sheet.

[2] David Riddle, Mathisen Way, Poyal Road, Colnbrook, Slough, Danger and Detection of hydrogen sulphide gas in oil and Gas exploration and production. April/May 2009

[3] Hydrogen Sulfide, Oil and Gas, and People's Health By Lana Skrtic, UC Berkley.

[4] Effects of Electronic Charge Transfer between adsorbate and Solid on Chemisorption and Catalysis P.B. Weisz,Socony- Vaccum laboratories,NewJersy.

[5] Synthesis of tungsten oxide nanowires Zongwen Liu, Yoshio Bando and Chengchun Tang. Advanced material laboratory, NIMS, Namiki 1-1, Tsukuba, Ibaraki 305-0044, Japan.

[6] Electrospinning of Nanofibres Dan Li and Younan Xia Advanced Materials, Vol 16, Issue 14, 2 Aug 2004.

[7] Large scale synthesis of tungsten oxide nanofibres by electrospinning Xiaofeng Lu, Xincai Liu, Wanjin Zhang, Ce Wang, Yen Wei Journal of Colloid interface Science 298(2006)996-999

[8] $\mathrm{H}_{2} \mathrm{~S}$ sensing properties of noble metal doped $\mathrm{WO}_{3}$ thin film sensor fabricated by micromachining Wei-Han Tao, Ching-Hsiang Tsai Sensors and actuators B 81 (2002) 237-247 